\title{
Localised blood-retinal barrier leakage and retinal light sensitivity in diabetic retinopathy
}

\author{
Toke Bek, Henrik Lund-Andersen
}

\begin{abstract}
Twenty patients with insulin dependent diabetes mellitus were selected on the basis of morphological signs of blood-retinal barrier leakage - namely, hard exudates seen on fundus photographs and/or localised leakage of fluorescein seen on fluorescein angiograms. Computerised perimetry was carried out in visual field areas that corresponded to the morphological lesions, and the visual field data were accurately correlated with the morphology as seen on fundus photographs and fluorescein angiograms. In addition, in seven of the patients who represented the range of leakage among the patients studied, the blood-retinal barrier leakage was quantitated by vitreous fluorophotometry. In 16 cases normal light sensitivity was found in retinal areas showing localised leakage as studied on fluorescein angiograms. In four cases with pronounced maculopathy, where scotomata occurred, there was no topographical correlation between the scotomata and barrier leakage. Furthermore hard exudates often, but not consistently, caused localised scotomata when arranged in dense conglomerates. The permeability values correlated with angiographically observed hyperfluorescence in the macular area. On the
\end{abstract}

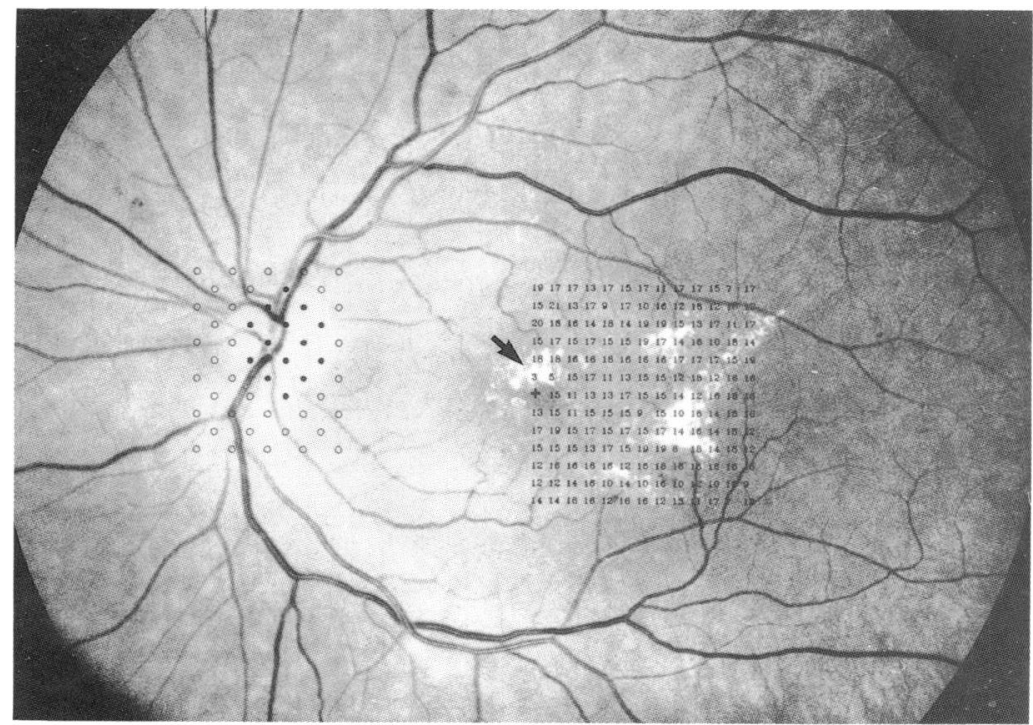

Figure 1A: Fundus photograph of a patient with formations of hard exudates in the macular area. The stimulus pattern extended from degree co-ordinates: $(x, y)=(0,-6)$ to $(x, y)=(12,6)$ The little conglomerate of hard exudates located over the point of fixation (arrow) is seen to cause a relative scotoma in the visual field. basis of the techniques employed in the present study it seems that breakdown of the blood-retinal barrier is an earlier event than disturbance of neurosensory function in the development of diabetic retinopathy. However, the findings give no evidence of a causal relationship between barrier leakage and damage to sensory cell function.

Diabetic retinopathy displays a complex morphological picture which results from retinal ischaemia, new vessel formation, and breakdown of the blood-retinal barrier.' Some studies have suggested that breakdown of the bloodretinal barrier leakage is an early, or perhaps the earliest, sign of pathology in the development of retinopathy. ${ }^{12}$ This barrier leakage can be studied qualitatively on fluorescein angiograms ${ }^{3}$ by detecting localised hyperfluorescence that increases and diffuses through the angiographic phases, and signs of barrier leakage can be seen on fundus photographs as hard exudates, which are assumed to be precipitates of lipoprotein exuded from the blood stream. Furthermore, a quantitative estimate of blood-retinal barrier leakage can be obtained by vitreous fluorophotometry.

Some authors have alternatively proposed that the earliest sign of pathology in diabetic retinopathy might be a disturbance of neurosensory function. ${ }^{46}$ Although pronounced fluorescein leakage in the macular area as studied on fluorescein angiograms is often associated with decreased visual acuity, the association between barrier leakage and neurosensory function has not been examined in detail.

The purpose of the present study was to correlate signs of blood-retinal barrier leakage as seen on fundus photographs and fluorescein angiograms with neurosensory function as determined by computerised perimetry. To support the interpretation of the angiograms, vitreous fluorophotometry was done in seven patients who represented the range of leakage among the patients studied.

\section{Materials and methods}

Twenty patients with insulin dependent diabetes mellitus and with signs of blood-retinal barrier leakage - namely, hard exudates seen on fundus 
photographs and/or localised leakage of fluorescein seen on fluorescein angiograms - were included. They had a routine ophthalmological examination including visual acuity, slit-lamp examination, tonometry, ophthalmoscopy, fluorescein angiography, and computerised perimetry. In addition vitreous fluorophotometry was carried out in seven of the patients. Informed consent was obtained.

The ametropia of the eyes ranged between $-1.75 \mathrm{D}$ and $+4.5 \mathrm{D}$, and the most severe astigmatism was $1.25 \mathrm{DC}$. In four patients with pronounced maculopathy the best corrected visual acuity was 6/12-6/18. In all the other patients the visual acuity was 6/6. Except for funduscopic signs of retinopathy the other findings on ophthalmic examination were normal in all the patients.

\section{PHOTOGRAPHY}

Photography was performed with a Canon CF$60 \mathrm{Z}$ fundus camera employing Ektachrome 64 (EPR 135-36) film for fundus photography, and Ilford HP5 black-and-white film for fluorescein angiography. $5 \mathrm{ml} 10 \%$ fluorescein sodium was injected into an antecubital vein for fluorescein angiography and vitreous fluorophotometry. Angiograms were taken of the central fundus in fast sequence during the filling phase of the vessels, and with regular intervals until $15 \mathrm{~min}$ after injection. Prior to photography cycloplegia and mydriasis were induced with metaoxedrine $10 \%$ and tropicamide $1 \%$ eyedrops.

\section{PERIMETRY}

Perimetry was carried out with a Humphrey Field Analyzer (HFA). Full-threshold examinations were performed in a stimulus pattern measuring $12^{\circ} \times 12^{\circ}$ with a density of $1^{\circ}$, Goldmann stimulus size I being employed. Since the minimum density of customised (individually defined) stimulus patterns on the HFA is $2^{\circ}$, four intersecting patterns with a displacement of $1^{\circ}$ in the vertical and the horizontal plane were subsequently tested, and finally merged. The stimulus patterns were placed in areas of the
Figure 1B: Fluorescein angiogram from the same patient 592 s after intravenous injection of fluorescein. It can be seen that the decibel values located over the are displaying leakage do no differ from values located over normally appearing retina. Visual acuity was 6/6. Permeability: $2 \cdot 2 \times$ $10^{-7} \mathrm{~cm} / \mathrm{s}$. visual field that approximately corresponded to the retinal areas where morphological lesions had been observed by prior funduscopic examination. Threshold values that deviated more than $5 \mathrm{~dB}$ from the expected value calculated on basis of answers from adjoining points were retested (standard procedure on the HFA). The blind spot was delimited with Goldmann stimulus size II with a single intensity of $10 \mathrm{~dB}$ in two special point patterns located in the area of the blind spot, one (pattern A) extending from degree coordinates $(x, y)=(11,-7)$ to $(19,3)$ and one (pattern B) from degree co-ordinates $(x, y)=$ $(12,-8)$ to $(18,2)$. By merging these two patterns, a spatial resolution of $1.4^{\circ}$ could be obtained in this area. All patients could see the perimeter screen sharply during perimetry without the addition of a corrective lens.

For control of baseline decibel values with Goldmann stimulus size I, 10 normal persons (volunteers), aged 25-56, with no prior history of ophthalmological disease were tested in one eye with full-threshold strategy employing programme 30-2 on the HFA. Decibel values in each stimulus point were averaged.

Fixation was continually checked on the fixation monitor supplied on the HFA, which displays the eye on a video screen during perimetry. Fixation was stable during all test sessions in all the patients and normal persons included.

\section{SUPERIMPOSITION TECHNIQUE}

The superimposition of visual field data on to fundus pictures was carried out according to a technique recently developed in our laboratory. ${ }^{7}$ This technique is applicable to a fundus photograph (subtending approximately $60^{\circ}$ with the camera employed here) with the foveal region in centre, and the accuracy of the overlay of visual field data on to the corresponding fundus photograph is better than $1^{\circ}$.

The technique was developed by establishing imaging in the fundus camera in an optical model. This model consisted of a concave spherical surface containing targets with known angular position and a lens in front of the sphere. By photographing the sphere through a $+3 \mathrm{D}$ lens located $0.33 \mathrm{~m}$ in front of (in the centre of) the sphere, imaging through the fundus camera when photographing the fundus of an emmetropic eye was simulated. By studying the location on the film of targets with known angular position in the sphere, camera distortion could be established. By subsequently varying the power of the lens and focusing the sphere through the lens, imaging in the fundus camera when photographing eyes with different degrees of ametropia was simulated. On photographs of the sphere the size of the photographic field of view as a function of ametropia could thereby be established.

With this knowledge of the optical imaging in the fundus camera, the visual field printout could be reduced in each patient to match the size of the corresponding fundus photograph. The visual field was inverted about a horizontal axis, and the overlay was subsequently done on a basis of two points of reference. One, the fixation 


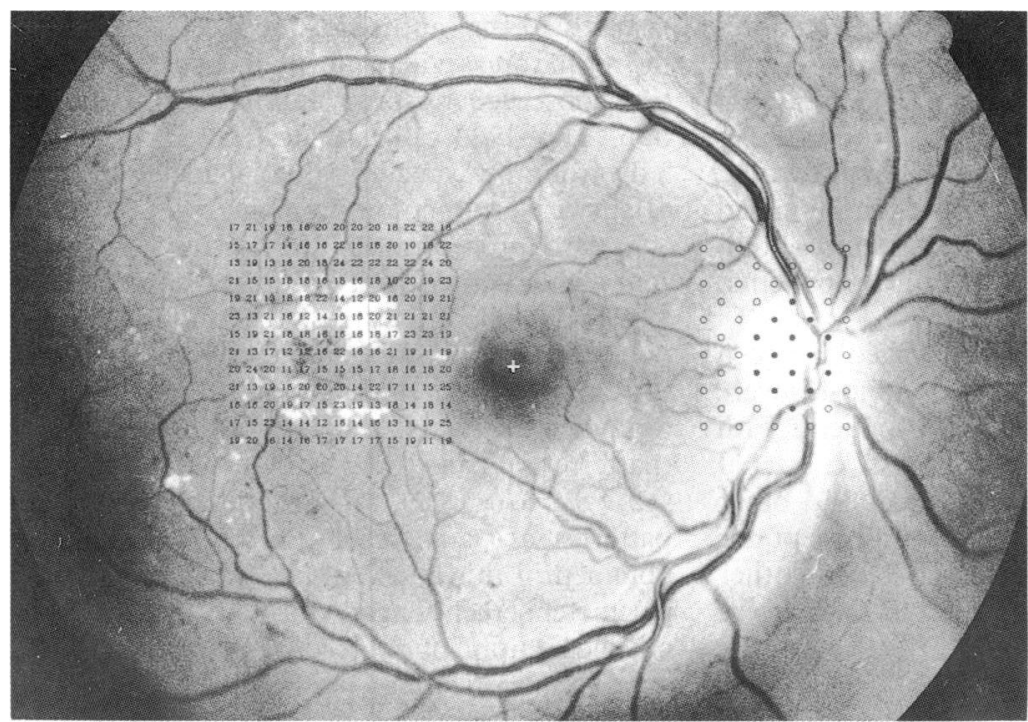

Figure 2A: Fundus photograph of a patient with circinate conglomerates of hard exudates temporal to the foveola. The stimulus pattern extended from degree co-ordinates $(x, y)=$

$(-16,-4)$ to $(x, y)=(-4,8)$

The exudates are seen not to cause any registrable decrease in retinal light sensitivity.

Figure 2B: Fluorescein angiogram of the same patient 405 s after intravenous injection of fluorescein. Localised retinal areas with pronounced leakage are seen to have normal light sensitivity. Visual acuity was 6/6. Permeability: $3 \cdot 8 \times 10-7$ $\mathrm{cm} / \mathrm{s}$.

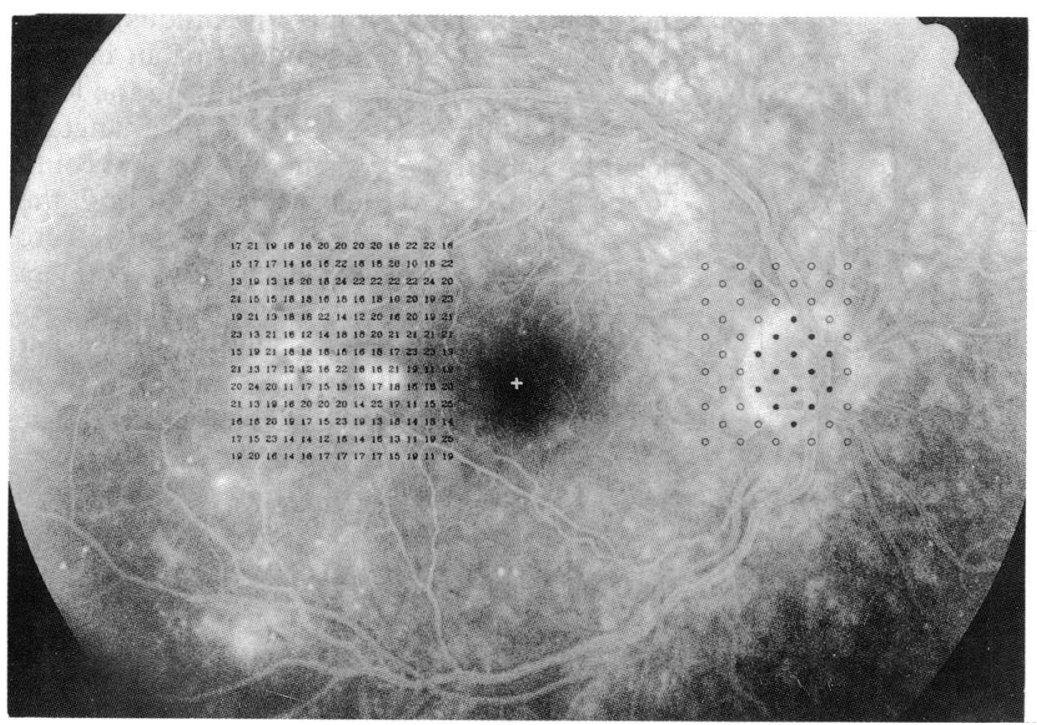
Vitreous fluorophotometry was carried out with the Fluorotron Master (Coherent, Palo Alto, California). Permeability values were calculated by relating the plasma time-integral of nonprotein bound fluorescein at the time of measurement (approximately $60 \mathrm{~min}$ ) to the fluorescein concentration profile in the vitreous body. The method is described in detail elsewhere. ${ }^{8}$

\section{Results}

In Figures 1 to 3 representative examples of visual field data correlated with corresponding fundus morphology are shown. In the point patterns used to delimit the blind spot closed circles indicate stimulus points not seen, whereas open circles indicate seen points. The fixation point is indicated with a cross. The numbers indicate decibel values obtained with Goldmann stimulus size $I$ in the individually placed stimulus patterns, and each decibel value in the pattern has been placed over the corresponding retinal area. For points tested twice (see 'Materials and methods' section) the average of the two decibel values was employed.

In the normal volunteers the average of decibel values from each stimulus point showed a decrease of $0.4 \mathrm{~dB} /$ degree from the centre towards the periphery of the visual field, with an extrapolated value of $21 \mathrm{~dB}$ in the centre and ranging $\pm 3 \mathrm{~dB}$ from this mean line. For all patients the decibel values in non-scotomatous areas were within the range of normal persons.

No correlation was found between bloodretinal barrier leakage and retinal light sensitivity. In 16 patients who represented all degrees of barrier leakage there was normal light sensitivity in areas which displayed localised leakage (see Figs 1 and 2). In four patients localised scotomata occurred. These patients had pronounced morphological signs of maculopathy, in three cases including macular oedema (see Fig 3 ), and when the scotomata included the foveola there was an accompanying decrease in visual acuity. However, these scotomata did not correlate topographically with barrier leakage as studied on fluorescein angiograms; this is, localised leaking areas as well as localised non-leaking areas occurred in the same pattern over both normally and abnormally functioning retina.

The permeability values obtained by vitreous fluorophotometry ranged between $1.2 \times 10^{-7}$ $\mathrm{cm} / \mathrm{s}$ and $7 \cdot 0 \times 10^{-7} \mathrm{~cm} / \mathrm{s}$ (mean normal value $\left.1 \cdot 1 \times 10^{-7} \mathrm{~cm} / \mathrm{s}(\mathrm{SD} 0.4) \times 10^{-7} \mathrm{~cm} / \mathrm{s}\right){ }^{8}$ There was a correlation between the permeability value and macular hyperfluorescence as assessed on pictures of the late fluorescein angiographic phases. Furthermore, hard exudates often, but not consistently, caused scotomata in the visual field when arranged in dense conglomerates.

\section{Discussion}

In the present study computerised perimetry was carried out in 20 insulin dependent diabetic patients with funduscopic signs of break-down of the blood-retinal barrier, and the visual field data were accurately correlated with the corresponding morphology seen on fundus photographs and fluorescein angiograms. No correlation was found between retinal light sensitivity as determined by computerised perimetry and localised blood-retinal barrier leakage as studied by fluorescein angiography. However, hard exudates, which are assumed to be precipitates of lipoprotein exuded from the blood stream, often caused localised scotomata when arranged in larger conglomerates.

In the literature much attention has been directed towards establishing the nature of the initial pathophysiological event in the development of diabetic retinopathy. Several different views have been proposed. One view suggests that the initial event is a breakdown of the bloodretinal barrier, ${ }^{12}$ and another view that it is a disturbance of neurosensory function caused by metabolic dysregulation. ${ }^{46}$

In the present study this question has been investigated by employing retinal light sensitivity as a measure of neurosensory function and leakage of fluorescein as a measure of blood- 


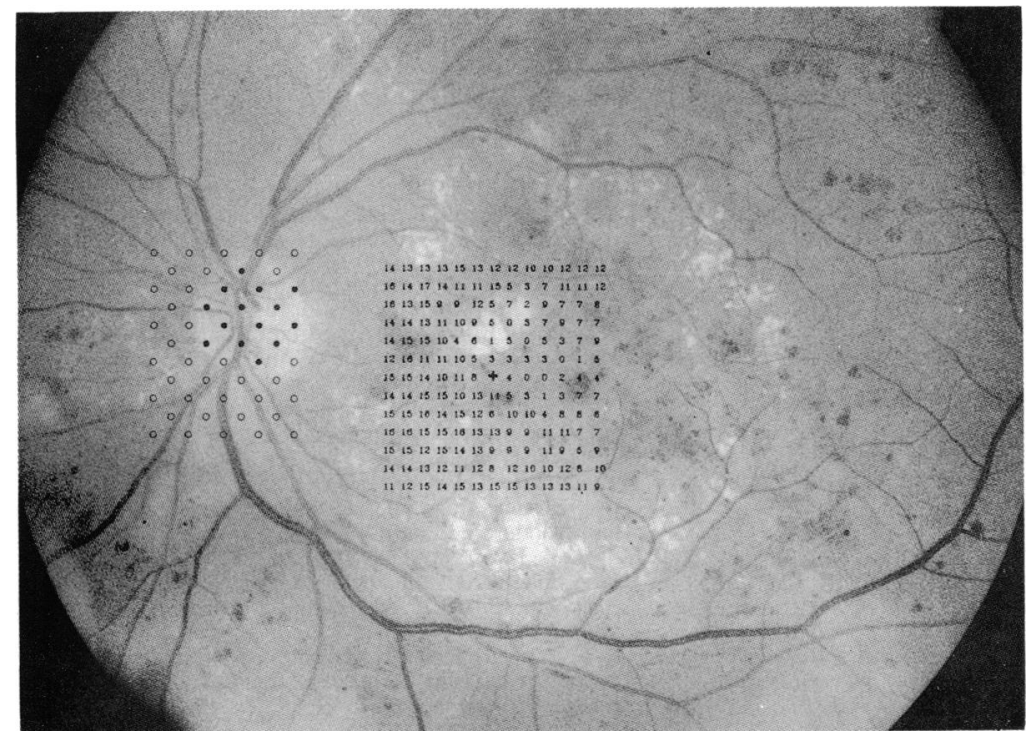

Figure 3A: Fundus photograph of a patient with pronounced diabetic maculopathy. The stimulus pattern extended from degree co-ordinates $(x, y)=$ $(-6,-6)$ to $(x, y)=(6,6)$. Retinal oedema was detected in the whole macular area by stereo microscopic fundus examination.

Figure 3B: Fluorescein angiogram of the same patient 569 s after intravenous injection of fluorescein. Pronounced spotwise and diffuse leakage is seen in the macular area. Visual acuity was $6 / 18$, and a central scotoma is seen to include the point of fixation. The retinal areas displaying abnormal function (low decibel values) cannot be distinguished from normally functioning retina on the basis of barrier leakage. The decibel values in the left part of the stimulus pattern are normal. Vitreous

fluorophotometry was not done in this patient. retinal barrier breakdown. Blood-retinal barrier leakage was defined as localised hyperfluorescence that increased and diffused through the angiographic phases. The validity of this criterion was supported by the fact that in seven patients the permeability values obtained by vitreous fluorophotometry (referring only to leakage in the macular area ${ }^{9}$ ) correlated with the angiographically observed macular hyperfluorescence. In 16 patients who represented all degrees of barrier leakage there was normal light sensitivity in the areas displaying localised leakage, and in four patients with pronounced maculopathy where localised scotomata occurred no correspondence was found between the scotomata and barrier leakage as studied on fluorescein angiograms. Thus, if the techniques used are suitable for elucidating this question, it seems that the breakdown of the blood-retinal barrier is an earlier event than disturbance of neurosensory function in the development of diabetic retinopathy. However, since no topographical correlation was found between barrier leakage and decreased light sensitivity in any of the patients studied, the findings give no evidence of a causal relationship between barrier leakage and damage to sensory cell function. For a more certain statement about lack of causality

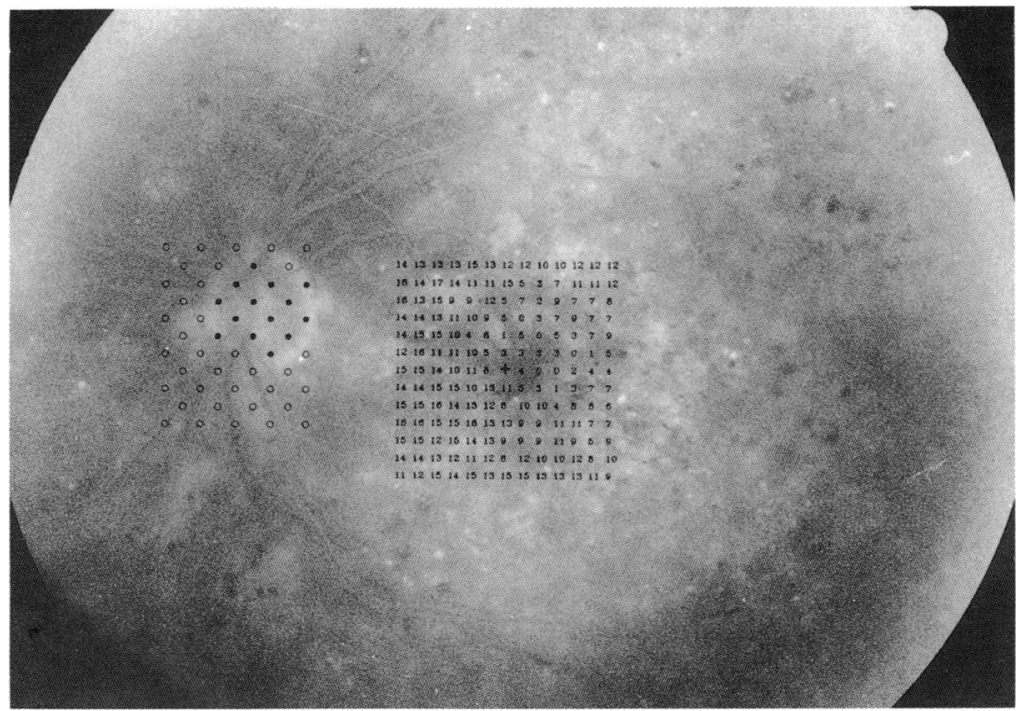

to be made, however, a prospective study is needed in which barrier status is followed in retinal areas that later give rise to scotomata.

It is likely that tissue damage caused by retinal oedema may have contributed to impairment of sensory cell function in some of the cases where scotomata occurred. However, the relation of retinal oedema to blood-retinal barrier leakage is rather difficult to evaluate, since oedema reflects the sum of net transcapillary fluid flow and production of metabolic water in the retinal tissue, whereas barrier leakage studied with fluorescein angiography and vitreous fluorophotometry almost exclusively refers to leakage out of the blood vessels.

The blood-retinal barrier system is composed of two barriers - an inner barrier consisting of the endothelial lining of the retinal capillaries, and an outer barrier consisting of the pigment epithelium. A differentiation of these two barriers is not possible by merely studying leakage on fluorescein angiograms. Leakage through each of these two barriers may indicate very different pathophysiological mechanisms for the inner barrier a disturbance of the retinal vascular system, and for the outer barrier a disturbance of the pigment epithelium, which is supplied by the choroidal cirulatory system. Possible neuronal damage caused by breakdown of the two barriers might in theory be differentiated by determination of localised neurosensory function, for example, by employing computerised perimetry. Leakage through the outer barrier would be a consequence of disturbed function of the pigment epithelium and therefore possibly be accompanied by an impaired function of the sensory cells, eventually resulting in scotomata in the visual field. In contrast, leakage through the inner barrier would not primarily cause damage to retinal neurons and therefore, at least in the initial stages, not cause scotomata in the visual field. Provided such reasoning is valid, the findings of the present study seem to indicate that blood-retinal barrier leakage is mainly caused by a breakdown of the inner barrier. However, a technique for the true separation of the two barriers is needed in order to clarify this question.

When studying retinal light sensitivity in areas displaying localised blood-retinal barrier leakage the scotomata to be found, if any, must be expected to be small. Therefore appropriate stimulus conditions for the detection of small scotomata are required. In a recent study it has been established that the smallest stimulus size possible should be employed when dense stimulus patterns are used for the detection of small scotomata. ${ }^{10}$ Thus the optimal stimulus parameters available on the HFA were chosen by combining Goldmann stimulus size I (subtending approximately $0 \cdot 1^{\circ}$ ) with a stimulus density of $1^{\circ}$. It is evident that even with these stimulus parameters smaller lesions may easily have escaped detection. This may explain why isolated hard exudates did not result in a registrable decrease in retinal light sensitivity. However, some larger conglomerates of hard exudates also failed to cause scotomata, which would be expected from their size. Most probably the morphological entity of a hard exudate 
as seen by funduscopic examination covers lesions with a wide range of thickness and biochemical composition, and therefore also a wide range of translucency.

In conclusion, the findings of the present study seem to indicate that breakdown of the blood-retinal barrier is an earlier event than disturbance of neurosensory function in the development of diabetic retinopathy. However, the findings provide no evidence of a causal relationship between barrier leakage and damage to sensory cell function.

This study was supported by Mimi and Victor Larsen's Foundation, the John and Birthe Meyer Foundation, and Carl Larsen's Foundation.

The skilful assistance of photographer Hans Henrik Petersen and technician Bente Mertz is gratefully acknowledged.

1 Ashton N. Pathogenesis of diabetic retinopathy. In: Little HL, Jack RL, Patz A, Forsham PH, eds. Diabetic retinopathy. New York: Thieme-Stratton, 1983: 85-106.
2 Cunha-Vaz J, Faria De Abreu JR, Campos AJ, Figo GM. Early breakdown of the blood-retinal barrier in diabetes. BrF Ophthalmol 1975; 59: 649-56.

3 Bresnick GH, Segal P, Mattson D. Fluorescein angiographic and clinicopathologic findings. In: Little HL, Jack RL Patz A Forsham PH, eds Diabetic retinopathy. New York: Thieme-Stratton, 1983: 37-71.

4 Bloom A, Heath JH, Kelsey PR, Hunter PR, Bridgden WD. The use of the Roth-Keeler central field scotometer in the 73. 73. disorder. Arch Ophthalmol 1986; 104: 989-90.

ERG in diabetics. In: Francos J. The clinical imonsen SE. ERG in diabetics. In: François J, ed. The clinical value of electroretinography. Fifth ISCERG Symposium,

7 Bek T, Lund-Andersen H. Accurate superimposition of visual field data onto fundus photographs. Acta Ophthalmol (Kbh) 1990; 68: 11-8.

8 Lund-Andersen H, Krogsaa B, la Cour M, Larsen J. Quantitative vitreous fluorophotometry applying a mathematical model of the eye. Invest Ophthalmol Vis Sci 1985; 5: 698-710.

9 Dalgaard $P$, Lund-Andersen $H$, Barker V. Vitreous Fluorophotometry: mathematical analysis of the effect of peripheral leakage on axial scans. Invest Ophthalmol Vis Sci 1989; 30: 1522-6.

10 Bek T, Lund-Andersen $H$. The influence of stimulus size on perimetric detection of small scotomata. Graefes Arch Clin Exp Ophthalmol 1989; 227: 531-4. 